\title{
Neutrino masses and their ordering: Global Data, Priors and Models
}

\author{
S. Gariazzo, ${ }^{a}$ M. Archidiacono, ${ }^{b}$ P.F. de Salas, ${ }^{a}$ O. Mena, ${ }^{a}$ C.A. \\ Ternes, ${ }^{a}$ and M. Tórtola ${ }^{a}$
}

${ }^{a}$ Instituto de Física Corpuscular (CSIC-Universitat de València)

Parc Científic UV, C/ Catedrático José Beltrán, 2

E-46980 Paterna (Valencia), Spain

${ }^{b}$ Institute for Theoretical Particle Physics and Cosmology (TTK)

RWTH Aachen University, D-52056 Aachen, Germany

E-mail: gariazzo@ific.uv.es, archidiacono@physik.rwth-aachen.de, pabferde@ific.uv.es, omena@ific.uv.es, chternes@ific.uv.es, mariam@ific.uv.es

\begin{abstract}
We present a full Bayesian analysis of the combination of current neutrino oscillation, neutrinoless double beta decay and Cosmic Microwave Background observations. Our major goal is to carefully investigate the possibility to single out one neutrino mass ordering, namely Normal Ordering or Inverted Ordering, with current data. Two possible parametrizations (three neutrino masses versus the lightest neutrino mass plus the two oscillation mass splittings) and priors (linear versus logarithmic) are exhaustively examined. We find that the preference for NO is only driven by neutrino oscillation data. Moreover, the values of the Bayes factor indicate that the evidence for NO is strong only when the scan is performed over the three neutrino masses with logarithmic priors; for every other combination of parameterization and prior, the preference for NO is only weak. As a by-product of our Bayesian analyses, we are able to (a) compare the Bayesian bounds on the neutrino mixing parameters to those obtained by means of frequentist approaches, finding a very good agreement; (b) determine that the lightest neutrino mass plus the two mass splittings parametrization, motivated by the physical observables, is strongly preferred over the three neutrino mass eigenstates scan and (c) find that logarithmic priors guarantee a weakly-to-moderately more efficient sampling of the parameter space. These results establish the optimal strategy to successfully explore the neutrino parameter space, based on the use of the oscillation mass splittings and a logarithmic prior on the lightest neutrino mass, when combining neutrino oscillation data with cosmology and neutrinoless double beta decay. We also show that the limits on the total neutrino mass $\sum m_{\nu}$ can change dramatically when moving from one prior to the other. These results have profound implications for future studies on the neutrino mass ordering, as they crucially state the need for self-consistent analyses which explore the best parametrization and priors, without combining results that involve different assumptions.
\end{abstract}




\section{Contents}

1 Introduction $\quad 1$

2 Method and data 3

2.1 Statistical analysis 3

2.2 Parametrizations 4

$\begin{array}{lll}3 & \text { Experimental data } & 7\end{array}$

3.1 Neutrino oscillation data $\quad 7$

3.2 Neutrinoless double-beta decay data 9

3.3 Cosmological data 9

4 Results $\quad 10$

4.1 Global Bayesian constraints on the neutrino mixing parameters 10

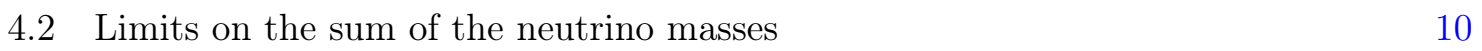

4.3 Implications for the mass ordering 13

5 Conclusions

\section{Introduction}

Neutrinos are light elementary particles which exclusively interact via weak interactions, and therefore they decouple from the thermal bath in the early Universe as extremely relativistic states, constituting a hot dark matter component in our Universe. Earth-based experiments have demonstrated that neutrinos oscillate $[1,2]$ and therefore that neutrinos are massive particles, implying the first departure from the Standard Model (SM) of Particle Physics. Neutrinos, as hot dark matter particles, possess large thermal velocities ${ }^{1}$, cluster only at scales below their free streaming scale and consequently do not contribute to structure formation at small scales [3-5]. The Cosmic Microwave Background (CMB) is also affected by the presence of massive neutrinos, as these particles may turn non-relativistic around the time of photon decoupling. Since neutrino oscillation physics is only sensitive to the squared mass differences $\left(\Delta m_{i j}^{2}=m_{i}^{2}-m_{j}^{2}\right)$, we cannot reliably compute the contribution of relic neutrinos to the (hot) dark matter of the Universe until we establish the absolute scale of neutrino masses. Current oscillation data can be remarkably well-fitted in terms of two squared mass differences, dubbed as the solar mass splitting $\left(\Delta m_{21}^{2} \simeq 7.6 \times 10^{-5} \mathrm{eV}^{2}\right)$ and the atmospheric mass splitting $\left(\left|\Delta m_{31}^{2}\right| \simeq 2.5 \times 10^{-3} \mathrm{eV}^{2}\right)[6]$. Thanks to matter effects in the Sun, we know that $\Delta m_{21}^{2}>0 .^{2}$ Since the atmospheric mass splitting $\Delta m_{31}^{2}$ is currently measured only via neutrino oscillations in vacuum, which exclusively depend on its absolute value, its sign is unknown at the moment. As a consequence, we have two possibilities for the ordering of

\footnotetext{
${ }^{1} \mathrm{~A}$ rough estimate of the thermal velocity of the neutrino relics with $1 \mathrm{eV}$ masses is $100 \mathrm{~km} / \mathrm{s}$, which is comparable to the typical velocity dispersion of a galaxy. Dwarf galaxies have much smaller velocity dispersions, implying that neutrinos can not cluster to form the smaller structures we observe in our Universe. Cold dark matter instead has a negligible velocity dispersion, contributing to clustering at all scales.

${ }^{2}$ Note that the observation of matter effects in the Sun constrains the product $\Delta m_{21}^{2} \cos 2 \theta_{12}$ to be positive. Therefore, depending on the convention chosen to describe solar neutrino oscillations, matter effects either fix the sign of the solar mass splitting $\Delta m_{21}^{2}$ or the octant of the solar angle $\theta_{12}$, with $\Delta m_{21}^{2}$ positive by definition.
} 
neutrino masses, which can be normal $\left(\Delta m_{31}^{2}>0\right)$ or inverted $\left(\Delta m_{31}^{2}<0\right)$. Future terrestrial experiments aim at measuring the sign of $\Delta m_{31}^{2}$ exploiting the matter effects in Earth using long baseline accelerators [7] and atmospheric neutrino experiments [8, 9].

The measurement of the neutrino mass ordering, together with the determination of the amount of $\mathrm{CP}$ violation in the lepton sector and the extraction of the neutrino mass nature (Dirac versus Majorana) are mandatory steps to unravel the neutrino mass mechanism and the origin of neutrino masses. Neutrinoless double beta decay $(0 \nu \beta \beta)$, the rarest nuclear weak process in which the lepton number is violated by two units, could be realised in nature only if neutrinos are Majorana [10-12]. A positive detection of the so-called effective Majorana neutrino mass would not only firmly assess that neutrinos are Majorana particles, but could also potentially establish the neutrino mass ordering. This is also related to the fact that we can put constraints on the absolute scale of neutrino masses. Depending on the mass ordering, a lower bound on the sum of the three active neutrino masses $\left(\Sigma m_{\nu}\right)$ is established. In the Normal Ordering (NO) we have $\Sigma m_{\nu} \gtrsim 0.06 \mathrm{eV}$, while in the Inverted Ordering (IO) $\Sigma m_{\nu} \gtrsim 0.10 \mathrm{eV}$, where the exact numbers depend on the uncertainties on the squared mass differences. Should the experimental measurements on $\Sigma m_{\nu}$ be strong enough to rule out the parameter space corresponding to IO, we would know that the neutrino mass ordering must be normal. Cosmological studies can also be used to set upper bounds on $\Sigma m_{\nu}$ combining CMB data with different large scale structure observations, providing the bound $\Sigma m_{\nu}<0.12 \mathrm{eV}$ at 95\% C.L. [13-16]. The possible ways we can exploit to determine the neutrino mass ordering, therefore, include a) future oscillation facilities; b) future neutrinoless double beta decay facilities, which could have also the potential to unravel the hierarchical pattern [12, 17]; and c) next-generation of CMB and large scale structure surveys, which will notably improve the present cosmological limits on $\Sigma m_{\nu}[18,19]$ and consequently strongly constrain the IO case or even determine the mass ordering.

Last but not least, the current efforts on the development of an experiment devoted to the direct detection of relic neutrinos, the "PonTecorvo 3 Observatory for Light, Early-universe, Massive-neutrino Yield" (PTOLEMY) proposal [20], may lead to the determination of the absolute scale, the nature and the ordering of neutrino masses through a completely different way. PTOLEMY will use the mechanism of neutrino capture on $\beta$-decaying nuclei [21] in order to detect the small number of events due to the interaction of massive relic neutrinos with the $\sim 100 \mathrm{~g}$ of tritium in the detector. While the energy position in the electron spectrum of the peak due to relic neutrino scattering depends on the absolute scale of neutrino masses, the event rate may give information on the Dirac or Majorana nature of neutrinos, assuming that we will be able to properly compute the enhancement due to the clustering of relic neutrinos in the neighbourhood of the Milky Way [22, 23]. While the expected energy resolution of PTOLEMY will probably not allow to probe the non-degenerate case and distinguish the three different peaks in the electron spectrum due to the different neutrino mass eigenstates, which would provide us clean information on the mass ordering [24], the method will open the way for a future experiment to achieve this result.

Recently, plenty of work in the literature has been devoted to test whether a preference for one ordering over the other, given current data, exists. Namely, in Refs. [25-27], careful and dedicated analyses of current cosmological data, either alone or combined with oscillation measurements, have conservatively found Bayesian odds favouring normal versus inverted ordering at the level of $2: 1$ in [25] and $3: 2$ in [26], which indicate an extremely weak

\footnotetext{
${ }^{3}$ Since the current idea is to move the proposal to the Gran Sasso laboratories in Italy, the original name referring to the previous Princeton location has been recently changed.
} 
preference for the normal scheme. Also, a modest $2 \sigma$ preference was found by the frequentist approach of Ref. [27]. The authors of Ref. [28] agree with these findings, as they conclude that their Bayes factor between the normal and the inverted neutrino mass scenarios is not large enough to establish a clear preference for one ordering over the other.

By combining the results from the oscillation global fits carried out previously in Ref. [29] and the cosmological constraint $\Sigma m_{\nu}<0.13 \mathrm{eV}$ at $95 \%$ C.L. [13-16], adopting a different choice for the parametrization and the priors than those assumed in Refs. [25-27], the exercise of Ref. [30] resulted in a strong evidence for the normal ordering scenario, with odds $42: 1{ }^{4}$ This result was immediately debated in Ref. [31], where it was argued that the odds $42: 1$ are almost completely determined by the use of the logarithmic prior.

More recently, in Ref. [32], the authors considered neutrinoless double beta decay measurements [33-35] together with cosmological data [36] and oscillation results [29] and they found only very mild preference for the normal ordering, even if the assumed priors were also logarithmic (as those of Ref. [30]). In this case, however, the authors considered a different parametrization, based on the $\left(m_{\text {lightest }}, \Delta m_{21}^{2},\left|\Delta m_{31}^{2}\right|\right)$ parameters, which differs from the one addressed in Ref. [30], where the authors chose to scan over the three neutrino masses $\left(m_{1}, m_{2}, m_{3}\right)$.

The major goal of this study is to fully clarify the issue of the preference for one of the mass orderings which can be extracted from the current neutrino oscillation, cosmological and neutrinoless double beta decay measurements, describing how the assumptions on linear or logarithmic priors and on the two possible parametrizations described above can influence the results. Following this approach, we will ensure a self-consistent analysis and, consequently, self-consistent and reliable results, avoiding arbitrary assumptions and methods.

The structure of this manuscript is the following. In section 2 we describe the method that we adopt in our calculations: the Bayesian framework we are working with (2.1) and the adopted parametrizations (2.2). Section 3 is devoted to describe the experimental constraints we employ in our analysis, which include neutrino oscillation (3.1), neutrinoless double beta decay (3.2) and cosmological (3.3) data. In section 4 we review our results: the Bayesian constraints on the mixing parameters compared with the frequentist results (4.1), the limits on the sum of the neutrino masses (4.2) and the perspectives for the mass ordering determination (4.3). In section 5 we draw our conclusions.

\section{Method and data}

\section{$2.1 \quad$ Statistical analysis}

In the following, we shall briefly summarise the statistical analysis carried out here, based on a full Bayesian approach. For an extensive and illustrative discussion of the application of Bayesian methods in cosmology, we refer the reader to the review presented in Ref. [37]. Our major aim here is to test the two possible neutrino mass scenarios by means of model selection techniques. In the Bayesian framework, this translates into the calculation of the Bayesian evidence $Z$, also called the marginal likelihood, defined as the average of the likelihood $p(d \mid \theta, \mathcal{M})$ under a prior $p(\theta \mid \mathcal{M})$, for a specific model $\mathcal{M}$, a set of parameters $\theta$ and the dataset $d[37]$ :

$$
Z=p(d \mid \mathcal{M})=\int_{\Omega_{\mathcal{M}}} p(d \mid \theta, \mathcal{M}) p(\theta \mid \mathcal{M}) d \theta .
$$

\footnotetext{
${ }^{4}$ It is interesting to note that the same result, using our version of the Jeffreys' scale (see table 1), would be marked as "moderate".
} 


\begin{tabular}{c|c|c}
\hline$\left|\ln B_{\mathrm{NO}, \mathrm{IO}}\right|$ & Odds & strength of evidence \\
\hline$<1.0$ & $\lesssim 3: 1$ & inconclusive \\
$\in[1.0,2.5]$ & $(3-12): 1$ & weak \\
$\in[2.5,5.0]$ & $(12-150): 1$ & moderate \\
$>5.0$ & $>150: 1$ & strong \\
\hline
\end{tabular}

Table 1. Jeffreys' scale for estimating the strength of the preference for one model over the other (from Ref. [37]) when performing Bayesian model comparison analysis.

Applying Bayes' theorem, one can obtain the model posterior probability:

$$
p(\mathcal{M} \mid d) \propto p(\mathcal{M}) p(d \mid \mathcal{M}),
$$

where $p(\mathcal{M})$ is the prior probability associated to the model under consideration. In our analysis we will compare the models corresponding to the normal and inverted mass orderings, named $\mathcal{M}_{\mathrm{NO}}$ and $\mathcal{M}_{\mathrm{IO}}$, to which we assign identical prior probabilities $p\left(\mathcal{M}_{\mathrm{NO}}\right)=p\left(\mathcal{M}_{\mathrm{IO}}\right)=$ 0.5. When comparing the two given models, the quantity we are interested in is the ratio of the posterior probabilities, given by:

$$
\frac{p\left(\mathcal{M}_{\mathrm{NO}} \mid d\right)}{p\left(\mathcal{M}_{\mathrm{IO}} \mid d\right)}=B_{\mathrm{NO}, \mathrm{IO}} \frac{p\left(\mathcal{M}_{\mathrm{NO}}\right)}{p\left(\mathcal{M}_{\mathrm{IO}}\right)},
$$

where the quantity $B_{\mathrm{NO}, \mathrm{IO}}$ is the well-known Bayes factor, i.e. the ratio of the evidences of the two models:

$$
B_{\mathrm{NO}, \mathrm{IO}}=\frac{Z_{\mathrm{NO}}}{Z_{\mathrm{IO}}} \quad \Longrightarrow \quad \ln B_{\mathrm{NO}, \mathrm{IO}}=\ln Z_{\mathrm{NO}}-\ln Z_{\mathrm{IO}} .
$$

If the Bayes factor is larger than 1 ( $\ln B_{\mathrm{NO} \text {,IO }}>0$ ), observations favour $\mathcal{M}_{\mathrm{NO}}$ versus $\mathcal{M}_{\mathrm{IO}}$. If $B_{\mathrm{NO}, \mathrm{IO}}<1\left(\ln B_{\mathrm{NO}, \mathrm{IO}}<0\right)$ instead, data would favour $\mathcal{M}_{\mathrm{IO}}$ versus $\mathcal{M}_{\mathrm{NO}}$. The strength of the preference for one of the competing models over the other is usually determined by means of the Jeffreys' scale (see Ref. [37] and references therein), shown in table 1.

In order to compute the Bayesian evidence, we use the PolyChord nested sampler [38, 39], which can be integrated in the publicly available CosmoMC code, that includes the Boltzmann equation solver CAMB [40, 41] for the computation of cosmological quantities. These are the tools required to compute the Bayes factor, which will allow for a proper model comparison of the normal versus the inverted neutrino mass ordering.

\subsection{Parametrizations}

In order to derive the joint constraints from neutrino oscillations, $0 \nu \beta \beta$ and cosmological measurements we explore a vast parameter space, which consists of up to sixteen physical parameters. To obtain robust conclusions we describe the neutrino masses under several different assumptions, as detailed below.

The first set of parameters which we assume all throughout the analysis are the typical six parameters related to the standard cosmology within a flat $\Lambda \mathrm{CDM}$ universe:

$$
\left\{\Omega_{\mathrm{b}} h^{2}, \Omega_{\mathrm{c}} h^{2}, \Theta_{s}, \tau, n_{s}, \log \left[10^{10} A_{s}\right]\right\} .
$$

They are the baryon $\Omega_{\mathrm{b}} h^{2}$ and the cold dark matter $\Omega_{\mathrm{c}} h^{2}$ energy densities, the ratio between the sound horizon and the angular diameter distance at decoupling $\Theta_{s}$, the reionization optical 


\begin{tabular}{c|c||c|c||c|c}
\multicolumn{2}{c||}{ Cosmological } & \multicolumn{2}{c||}{$0 \nu \beta \beta$} & \multicolumn{2}{c}{ Neutrino mixing } \\
\hline Parameter & Prior & Parameter & Prior & Parameter & Prior \\
\hline$\Omega_{b} h^{2}$ & $0.019-0.025$ & $\alpha_{2}$ & $0-2 \pi$ & $\sin ^{2} \theta_{12}$ & $0.1-0.6$ \\
$\Omega_{c} h^{2}$ & $0.095-0.145$ & $\alpha_{3}$ & $0-2 \pi$ & $\sin ^{2} \theta_{13}$ & $0.00-0.06$ \\
$\Theta_{s}$ & $1.03-1.05$ & $\mathcal{M}_{76}^{0 \nu} \mathrm{Ge}$ & $4.07-4.87$ & $\sin ^{2} \theta_{23}$ & $0.25-0.75$ \\
$\tau$ & $0.01-0.4$ & $\mathcal{M}_{136}^{0 \nu} \mathrm{Xe}$ & $2.74-3.45$ & & \\
$n_{s}$ & $0.885-1.04$ & & & & \\
$\log \left(10^{10} A_{s}\right)$ & $2.5-3.7$ & \multicolumn{3}{|c}{}
\end{tabular}

Table 2. Cosmological parameters, $0 \nu \beta \beta$ parameters and neutrino mixing angles used in the analysis, with the adopted priors.

depth $\tau$, the scalar spectral index $n_{s}$ and the amplitude $A_{s}$ of the primordial power spectrum. The priors adopted on these six parameters are reported in the left column of table 2.

Concerning the neutrino mixing parameters, there are three angles and one CP-violating phase $\delta$ in the PMNS neutrino mixing matrix [42]. Given that current neutrino oscillation measurements are unable to set strong constraints on the value of $\delta$, in our simulations we will ignore this parameter, which moreover does not affect the cosmological or $0 \nu \beta \beta$ observables. In any case, the global neutrino oscillation results adopted here are computed marginalising away the $\mathrm{CP}$ violating phase. Concerning the three mixing angles, we use the physical parameters $\sin ^{2} \theta_{12}, \sin ^{2} \theta_{13}$ and $\sin ^{2} \theta_{23}$, which are the ones directly involved in the three-neutrino framework oscillation probabilities, with the priors shown in the right column of table 2. The prior ranges were chosen to fully cover the $3 \sigma$ region around the best fit for both mass orderings.

Current bounds from $0 \nu \beta \beta$ experiments are provided in terms of the so-called effective Majorana mass of the electron neutrino:

$$
m_{\beta \beta}=\frac{m_{e}}{\mathcal{M}^{\prime \nu} \sqrt{G_{0 \nu} T_{1 / 2}^{0 \nu}}},
$$

where $T_{1 / 2}^{0 \nu}$ is the $0 \nu \beta \beta$ half-life, $m_{e}$ is the electron mass, $G_{0 \nu}$ is a phase-space factor and $\mathcal{M}^{\prime \nu}$ is the Nuclear Matrix Element (NME), a delicate and crucial quantity whose uncertainty can strongly affect the derived bounds on $m_{\beta \beta}$ from $0 \nu \beta \beta$ searches. Here we follow the approach of Ref. [32], which deals with the results reported by the Gerda [33], EXO-200 [34] and KamLAND-Zen [35] experiments. Therefore, we have two extra common parameters in our analyses, which encode the uncertainty on the NMEs of ${ }^{76} \mathrm{Ge}$ (in the case of Gerda) and ${ }^{136} \mathrm{Xe}$ (in the case of EXO and KamLAND-Zen). Following The Modest Proposal for the Ranges of Values of the NMEs provided by the authors of Ref. [43], we adopt the range [4.07 - 4.87] for ${ }^{76} \mathrm{Ge}$ and $[2.74-3.45]$ for ${ }^{136} \mathrm{Xe}$, as reported in the central column of table 2 . We have verified that using extended ranges for the NMEs does not affect significantly our results on the neutrino mass ordering. The other two extra parameters required for the $0 \nu \beta \beta$ analysis are related to the definition of the effective Majorana mass

$$
m_{\beta \beta}=\left|\sum_{k} e^{i \alpha_{k}} U_{\mathrm{PMNS}, \mathrm{ek}}^{2} m_{k}\right|,
$$

where $\alpha_{k}(k=1, \ldots, 3)$ are the Majorana phases, which play no role in neutrino oscillations 


\begin{tabular}{c|c|c||c|c|c}
\multicolumn{3}{c||}{ Case A } & \multicolumn{3}{c}{ Case B } \\
\hline Parameter & Prior & Range & Parameter & Prior & Range \\
\hline \hline$m_{1} / \mathrm{eV}$ & $\begin{array}{c}\text { inear } \\
\log \end{array}$ & $\begin{array}{c}0-1 \\
10^{-5}-1\end{array}$ & $m_{\text {lightest }} / \mathrm{eV}$ & $\begin{array}{c}\text { linear } \\
\log \end{array}$ & $\begin{array}{c}0-1 \\
10^{-5}-1\end{array}$ \\
\hline$m_{2} / \mathrm{eV}$ & $\begin{array}{c}\operatorname{linear} \\
\log \end{array}$ & $\begin{array}{c}0-1 \\
10^{-5}-1\end{array}$ & $\Delta m_{21}^{2} / \mathrm{eV}^{2}$ & $\operatorname{linear}$ & $5 \times 10^{-5}-10^{-4}$ \\
\hline$m_{3} / \mathrm{eV}$ & $\begin{array}{c}\operatorname{linear} \\
\log \end{array}$ & $\begin{array}{c}0-1 \\
10^{-5}-1\end{array}$ & $\left|\Delta m_{31}^{2}\right| / \mathrm{eV}^{2}$ & linear & $1.5 \times 10^{-3}-3.5 \times 10^{-3}$ \\
\hline
\end{tabular}

Table 3. Parametrizations of the neutrino masses and priors adopted in the analysis.

but are a basic ingredient in $0 \nu \beta \beta$ processes. ${ }^{5}$ One of the phases can always be rotated away, therefore we are left with two Majorana phases, which we choose to be $\alpha_{2}$ and $\alpha_{3}$. As done in previous related works $[17,26]$, we apply flat priors in the range $[0,2 \pi]$ for these two Majorana phases, whose values are totally unknown, as also reported in table 2 .

The latest parameters we need to account for are related to the description of neutrino masses. Even if the underlying theoretical model is the same, the physical parameter space can be described in different ways, which can be mapped one into the other using some (non-linear) transformations. While the physics does not change, different parameterizations can have different performances when comparing the theoretical model with the experimental data, which reflects in different Bayesian evidences. Here we distinguish two possible approaches, that we label as Case A and Case B. For the former one, Case A, we perform the scan over the individual neutrino masses (i.e. $m_{1}, m_{2}$ and $m_{3}$ ), following the approach of Refs. [26,30]. The latter one, Case B, focuses on the ( $\left.m_{\text {lightest }}, \Delta m_{21}^{2}, \Delta m_{31}^{2}\right)$ parameter space [32]. Being physically equivalent, the choice of one or the other parameterization basically reflects in different structures of the parameter space and of the prior function. Comparing Case A and Case B, therefore, can be seen as comparing the efficiency of different priors, non-linearly connected one to the other, in describing the available data. For both parametrizations we study linear and logarithmic priors on the physical mass parameters, in order to take into account that the true absolute mass scale is unknown, while we always use a linear prior for the squared mass differences. The complete list of priors is reported in table 3. Additionally, we will explore how variations in the prior ranges affect the final results. The comparison of the Bayesian evidences obtained with different priors and parametrizations will allow us to avoid results which are biased by subjective arbitrary choices in the analyses.

As we shall show in the following sections, given the current available measurements, the Bayesian analyses show that data prefer the informative priors of Case B over the general priors of Case A. In Case B, the priors are chosen to match the available information from neutrino oscillation experiments on the measured mass splittings and they ensure a very efficient scan in the parameter space. ${ }^{6}$ The situation is very similar to the one that we need to face when exploring the mixing angles: it is more efficient to scan the parameter space using the $\sin ^{2} \theta_{i j}$ quantities than using the mixing angles $\theta_{i j}$ themselves. While very futuristic galaxy and $21 \mathrm{~cm}$ surveys might be able to disentangle the individual values of the neutrino masses [44], present cosmological measurements are only sensitive to the total neutrino mass

\footnotetext{
${ }^{5}$ In the normal ordering case, some combinations of the Majorana phases could lead to an accidental cancellation of $m_{\beta \beta}$.

${ }^{6}$ The situation would be different without the information coming from the neutrino oscillation experiments, which severely constrain the relation between the three individual masses.
} 


\begin{tabular}{l||c|c|c} 
parameter & best fit $\pm 1 \sigma$ & $2 \sigma$ range & $3 \sigma$ range \\
\hline \hline$\Delta m_{21}^{2}\left[10^{-5} \mathrm{eV}^{2}\right]$ & $7.56 \pm 0.19$ & $7.20-7.95$ & $7.05-8.14$ \\
\hline$\left|\Delta m_{31}^{2}\right|\left[10^{-3} \mathrm{eV}^{2}\right](\mathrm{NO})$ & $2.55 \pm 0.04$ & $2.47-2.63$ & $2.43-2.67$ \\
$\left|\Delta m_{31}^{2}\right|\left[10^{-3} \mathrm{eV}^{2}\right](\mathrm{IO})$ & $2.47_{-0.05}^{+0.04}$ & $2.39-2.55$ & $2.34-2.59$ \\
\hline $\sin ^{2} \theta_{12} / 10^{-1}$ & $3.21_{-0.16}^{+0.18}$ & $2.89-3.59$ & $2.73-3.79$ \\
\hline $\sin ^{2} \theta_{23} / 10^{-1}(\mathrm{NO})$ & $4.30_{-0.18}^{+0.20} a$ & $3.98-4.78 \& 5.60-6.17$ & $3.84-6.35$ \\
$\sin ^{2} \theta_{23} / 10^{-1}(\mathrm{IO})$ & $5.98_{-0.15}^{+0.17} b$ & $4.09-4.42 \& 5.61-6.27$ & $3.89-4.88 \& 5.22-6.41$ \\
\hline $\sin ^{2} \theta_{13} / 10^{-2}(\mathrm{NO})$ & $2.155_{-0.075}^{+0.090}$ & $1.98-2.31$ & $1.89-2.39$ \\
$\sin ^{2} \theta_{13} / 10^{-2}(\mathrm{IO})$ & $2.155_{-0.092}^{+0.076}$ & $1.98-2.31$ & $1.90-2.39$ \\
\hline
\end{tabular}

Table 4. Neutrino oscillation parameters summary determined from the global analysis in [6]. The ranges for inverted ordering refer to the local minimum within this neutrino mass ordering.

\footnotetext{
${ }^{a}$ There is a local minimum in the second octant, at $\sin ^{2} \theta_{23}=0.596$ with $\Delta \chi^{2}=2.08$ with respect to the global minimum.

${ }^{b}$ There is a local minimum in the first octant, at $\sin ^{2} \theta_{23}=0.426$ with $\Delta \chi^{2}=3.0$ with respect to the global minimum for IO.
}

and the information on the three masses can be extracted only using the input from neutrino oscillation experiments. Since in Case A the parameter space is described using the three masses $m_{i}$, it is less efficiently explored than the parameter space of Case B, which includes the two squared mass differences.

\section{Experimental data}

\subsection{Neutrino oscillation data}

Our combined analysis is based on the global fit of neutrino oscillation parameters performed in Ref. [6]. For our Bayesian calculations, we adopt the $\chi_{\text {osc }}^{2}$ as a function of the mixing parameters and squared mass differences and we convert it into a likelihood $\mathcal{L}_{\text {osc }}$ using:

$$
\log \mathcal{L}_{\text {osc }}=-\chi_{\text {osc }}^{2} / 2 .
$$

Figure 1 and table 4 summarise the results of the global analysis [6]. To perform the global fit we have analysed data from all neutrino oscillation experiments. The parameters $\Delta m_{21}^{2}$ and $\sin ^{2} \theta_{12}$ are measured with great precision by the solar experiments [45-54] and the long baseline reactor experiment KamLAND [55]. The reactor neutrino data that we use comprise the 1230 days of data taken by Daya Bay [56], 500 live days of RENO data [57] and the Double Chooz far detector data from the far detector only-period (461 days) and the far and near detector-period (212 days) [58]. These short-baseline reactor experiments are mainly sensitive to $\sin ^{2} \theta_{13}$, whose determination in the global fit is dominated by the measurements of Daya Bay. Moreover, the most recent reactor results show a good sensitivity to the atmospheric mass splitting $\Delta m_{31}^{2}$ as well. The global fit to neutrino oscillations includes also atmospheric data from the phases I to III of the Super-Kamiokande experiment [59] and the neutrino telescopes IceCube DeepCore [60,61] and ANTARES [62]. Note, however, that the data and detector information needed to reproduce the last IceCube DeepCore results presented in [63] have not been made public yet, so those are not considered in our analysis. 

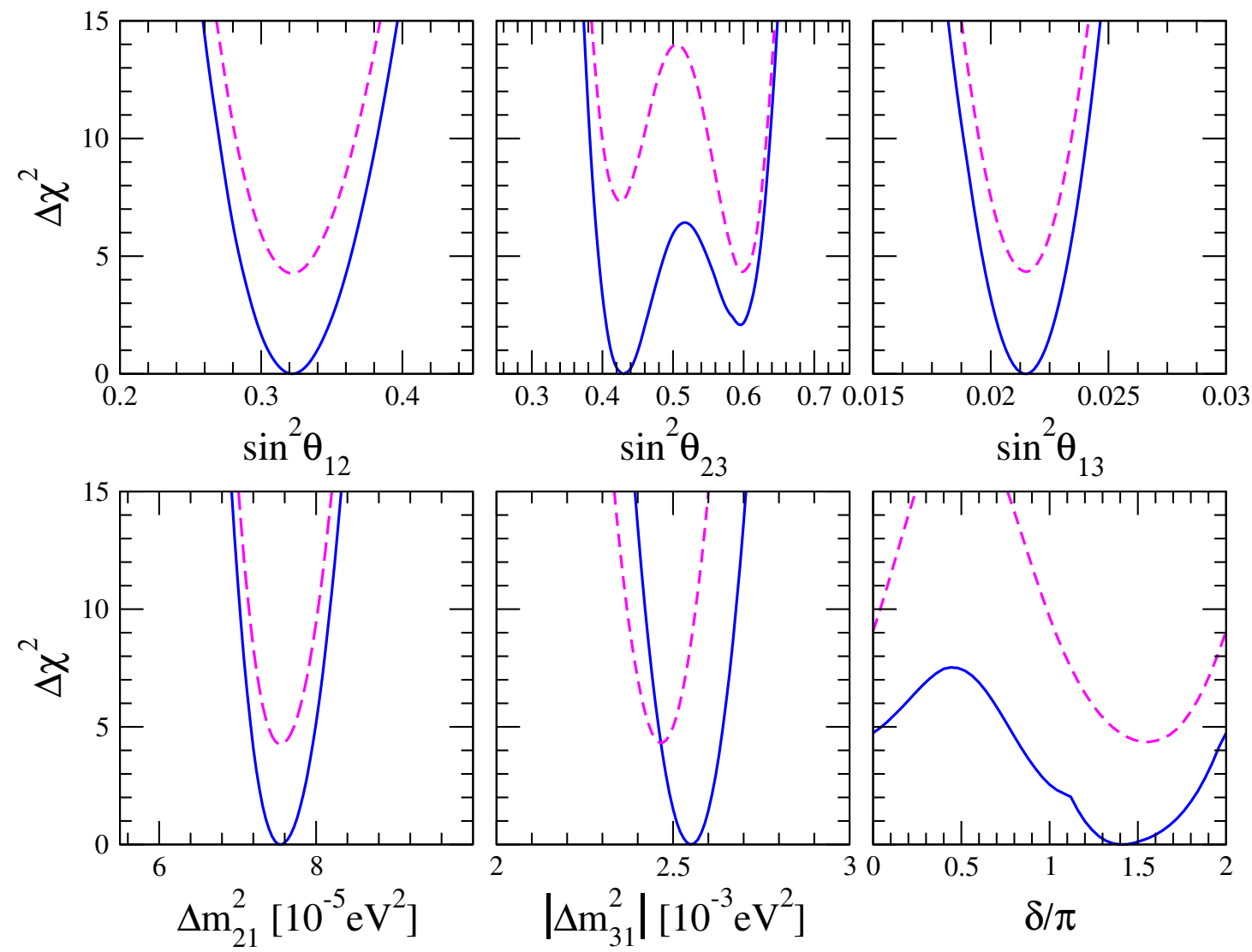

Figure 1. The profiles for the neutrino oscillation parameters. Solid blue lines correspond to NO and dashed magenta lines to IO.

We do not include the atmospheric neutrino results of Super Kamiokande phase IV [64] either, since there is not enough public information to reproduce the Super-Kamiokande analysis of atmospheric data outside the Collaboration. As it is explained in Ref. [6], neutrino telescopes start being competitive with long baseline experiments, although these still perform better when determining neutrino oscillation parameters. From long baseline accelerator neutrino experiments we include data taken by the already finished K2K [65] and MINOS experiments [66, 67], as well as from their successors, T2K [68, 69] and $\mathrm{NO} \nu \mathrm{A}[70,71]$. Besides constraining the atmospheric parameters $\Delta m_{31}^{2}$ and $\sin ^{2} \theta_{23}$, the long baseline experiments are also sensitive to $\sin ^{2} \theta_{13}$ and $\delta$. Indeed, most of the current sensitivity to the CP-violating phase $\delta$ comes from the combined analysis of T2K neutrino and antineutrino data. The current best fit value for this parameter tends towards maximal CP-violation, with $\delta=3 \pi / 2$. However, it remains the worst determined neutrino oscillation parameter, with most of the parameter space allowed at the $3 \sigma$ level. In any case, and as it was mentioned in section 2.2, this parameter is not crucial for the present analysis, so it has been marginalised over.

Another oscillation parameter that still has room for ambiguities is the atmospheric angle $\theta_{23}$, whose octant cannot yet be resolved at the $3 \sigma$ level. While T2K data prefer maximal mixing $\left(\sin ^{2} \theta_{23}=0.5\right)$, the MINOS and NO $\nu \mathrm{A}$ experiments show a preference for $\theta_{23}$ in the lower octant, resulting in a global best fit value of $\sin ^{2} \theta_{23}=0.43$. A local minimum is however present in the upper octant, with maximal mixing disfavoured at more than $2 \sigma$ confidence 
level, as can be seen in figure 1. When inverted mass ordering is assumed, a similar scenario is obtained, although in this occasion the global minimum lies in the upper octant and a local minimum for $\theta_{23}$ appears in the lower octant.

Comparing the two mass orderings with flavour oscillation data only, we obtain a slight preference for normal ordering with $\Delta \chi^{2}=4.3$. If we only consider the long baseline experiments $\mathrm{T} 2 \mathrm{~K}$ and $\mathrm{NO} \nu \mathrm{A}$, the difference between normal and inverted ordering is found to be $\Delta \chi^{2}=3.6$. This result is due to the better agreement between the $\mathrm{T} 2 \mathrm{~K}$ and $\mathrm{NO} \nu \mathrm{A}$ preferred values of $\sin ^{2} \theta_{23}$ in normal ordering with respect to inverted ordering. Adding the information from atmospheric data to the fit relaxes the tension between the preferred values of $\theta_{23}$ by $\mathrm{T} 2 \mathrm{~K}$ and $\mathrm{NO} \nu \mathrm{A}$ in $\mathrm{IO}$, to $\Delta \chi^{2}=3.1$. The best fit value for $\sin ^{2} \theta_{13}$ from the combined analysis of long baseline plus atmospheric data lies quite close to global best fit value, mainly fixed by the Daya Bay reactor experiment. However, in the case of normal ordering these two values are much closer to each other than for the inverted ordering case. Therefore, the inclusion of reactor data in the global neutrino fit increases again the preference for normal over inverted ordering to the final value of $\Delta \chi^{2}=4.3$. Further details on the determination of the mass ordering or the atmospheric angle octant from the global fit can be found in Ref. [6].

\subsection{Neutrinoless double-beta decay data}

We parameterize the constraints from $0 \nu \beta \beta$ searches following the same approach of Ref. [32]. Therefore, we use a combined likelihood

$$
\mathcal{L}_{0 \nu \beta \beta}=\prod_{i} \mathcal{L}_{0 \nu \beta \beta, i}
$$

where the terms $\mathcal{L}_{0 \nu \beta \beta, i}$ describe the likelihood for each experiment (Gerda [33], KamLANDZen [35] and EXO-200 [34]):

$$
\begin{aligned}
\mathcal{L}_{0 \nu \beta \beta, \operatorname{GERDA}}\left(T_{1 / 2}^{\mathrm{Ge}}\right) & \propto \exp \left[-\frac{\left(1 / T_{1 / 2}^{\mathrm{Ge}}+1.48\right)^{2}}{2 \times 0.461^{2}}\right], \\
\mathcal{L}_{0 \nu \beta \beta, \text { KamLAND-Zen phase I }}\left(T_{1 / 2}^{\mathrm{Xe}}\right) & \propto \exp \left[-\left(2.3 / T_{1 / 2}^{\mathrm{Xe}}+1.09 /\left(T_{1 / 2}^{\mathrm{Xe}}\right)^{2}\right)\right], \\
\mathcal{L}_{0 \nu \beta \beta, \text { KamLAND-Zen phase II }}\left(T_{1 / 2}^{\mathrm{Xe}}\right) & \propto \exp \left[-\left(9.71 / T_{1 / 2}^{\mathrm{Xe}}+28.1 /\left(T_{1 / 2}^{\mathrm{Xe}}\right)^{2}\right)\right], \\
\mathcal{L}_{0 \nu \beta \beta, \operatorname{EXO}}\left(T_{1 / 2}^{\mathrm{Xe}}\right) & \propto \exp \left[-\frac{\left(1 / T_{1 / 2}^{\mathrm{Xe}}-0.32\right)^{2}}{2 \times 0.30^{2}}\right]
\end{aligned}
$$

Here, the half-life decay times are given in units of $10^{25}$ years and all the parametrizations have been confirmed by the experimental collaborations [32]. The conversion between the half-life time and the neutrino masses is written in eqs. (2.6) and (2.7).

\subsection{Cosmological data}

Planck satellite measurements of the CMB temperature, polarization, and cross-correlation spectra from the 2015 release $[36,72]$ have been included. ${ }^{7}$ More precisely, we exploit both the high- $\ell(30 \leq \ell \leq 2508) T T$ and the low $\ell(2 \leq \ell \leq 29) T T$ likelihoods, based on the reconstructed $\mathrm{CMB}$ maps. The Planck polarization likelihood in the low-multipole regime $(2 \leq \ell \leq 29)$ is added to the previous two CMB datasets as well. Furthermore, we also

\footnotetext{
${ }^{7}$ We make use of the publicly available Planck likelihoods [73], see www.cosmos.esa.int/web/planck/pla.
} 


\begin{tabular}{c||c|c|c|c} 
parameter & bestfit & $1 \sigma$ range & $2 \sigma$ range & $3 \sigma$ range \\
\hline \hline$\Delta m_{21}^{2}\left[10^{-5} \mathrm{eV}^{2}\right]$ & 7.57 & $7.37-7.75$ & $7.21-7.96$ & $7.06-8.16$ \\
\hline$\left|\Delta m_{31}^{2}\right|\left[10^{-3} \mathrm{eV}^{2}\right](\mathrm{NO})$ & 2.55 & $2.51-2.59$ & $2.46-2.63$ & $2.42-2.67$ \\
$\left|\Delta m_{31}^{2}\right|\left[10^{-3} \mathrm{eV}^{2}\right](\mathrm{IO})$ & 2.46 & $2.42-2.50$ & $2.38-2.55$ & $2.34-2.59$ \\
\hline $\sin ^{2} \theta_{12}$ & 0.32 & $0.30-0.34$ & $0.29-0.36$ & $0.27-0.38$ \\
\hline $\sin ^{2} \theta_{23}(\mathrm{NO})$ & $0.43(0.59)$ & $0.40-0.47 \& 0.58-0.60$ & $0.38-0.50 \& 0.53-0.63$ & $0.37-0.65$ \\
$\sin ^{2} \theta_{23}(\mathrm{IO})$ & $0.60(0.43)$ & $0.56-0.63$ & $0.39-0.47 \& 0.54-0.65$ & $0.37-0.66$ \\
\hline $100 \sin ^{2} \theta_{13}(\mathrm{NO})$ & 2.14 & $2.06-2.22$ & $1.97-2.31$ & $1.89-2.39$ \\
$100 \sin ^{2} \theta_{13}(\mathrm{IO})$ & 2.15 & $2.07-2.24$ & $1.99-2.32$ & $1.90-2.41$ \\
\hline
\end{tabular}

Table 5. Bayesian results on the neutrino mixing parameters from the global fit of neutrino oscillation data. The results are in agreement with the corresponding frequentist global fit, see table 4, taken from ref. [6]. The parameters in parenthesis represent the $\sin ^{2} \theta_{23}$ local minima.

include here the high-multipole $(30 \leq \ell \leq 1996) E E$ and $T E$ likelihoods. All these likelihood functions have a non-negligible dependence on a given number of nuisance parameters (e.g. residual foreground contamination, calibration, and beam-leakage [36, 73]), which have also been taken into account and properly marginalised over.

\section{Results}

\subsection{Global Bayesian constraints on the neutrino mixing parameters}

Our model comparison analysis will provide, as a by-product, a Bayesian analysis of the neutrino oscillation parameters. These Bayesian limits can be compared with the frequentist analysis of Ref. [6]. Our results are summarised in table 5 and in figure 2. Notice that at the $3 \sigma$ level there is no significant difference with respect to the frequentist method (summarised in table 4 and figure 1), except for the atmospheric angle $\sin ^{2} \theta_{23}$. In the case of the frequentist approach, maximal mixing is excluded at $3 \sigma$ for the case of inverted ordering, while it remains allowed in the Bayesian analysis. In addition, the width of the confidence intervals for $\sin ^{2} \theta_{23}$ is larger in the Bayesian case than in the frequentist one for the two mass orderings. Note also that, in the Bayesian case, the local minimum found at $\sin ^{2} \theta_{23}=0.59$ for normal ordering now falls inside the $1 \sigma$ allowed range, contrary to what happens for inverted ordering or in the frequentist case for both orderings. To understand the differences between both analyses one should keep in mind that the marginalisation procedure in Bayesian parameter estimation is different from the one in the frequentist analysis. In the Bayesian case, the volume of the posterior distribution is also an important quantity, together with the relative difference in terms of $\chi^{2}$ (or, equivalently, in terms of the likelihood). Therefore, when performing Bayesian marginalisation, the posterior volume near the two minima (global and local) is large enough to prevent a strong exclusion of maximal atmospheric mixing in both mass orderings. On the other hand, since the local minimum in $\sin ^{2} \theta_{23}$ is closer to the global one in the NO case $\left(\Delta \chi^{2}=2.08\right)$ than in the IO one $\left(\Delta \chi^{2}=3.0\right)$, it still appears inside the $1 \sigma$ region for NO.

For the remaining mixing parameters, the allowed Bayesian intervals are in very good agreement with the frequentist ones, with only small deviations which are not significant.

\subsection{Limits on the sum of the neutrino masses}

Currently, the tightest constraints on the sum of the neutrino masses are provided by cosmological observations. In order to derive these bounds, the $\Lambda \mathrm{CDM}$ is typically assumed as 

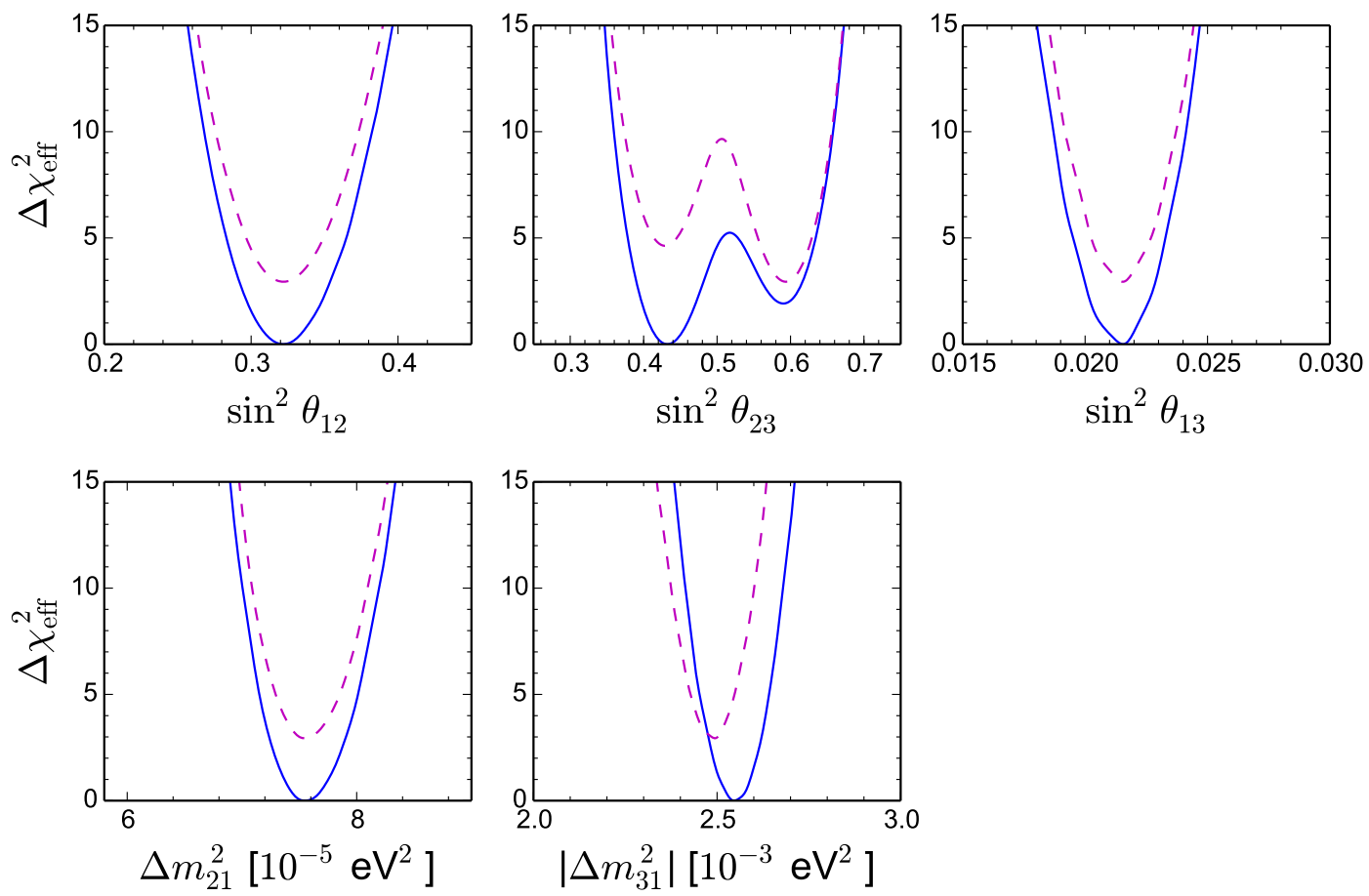

Figure 2. The profiles for the neutrino oscillation parameters as obtained from the Bayesian analysis. Blue (solid) lines correspond to NO and magenta (dashed) lines to IO. The effective $\chi^{2}$ is obtained from the posterior distribution function $p(x)$ for each parameter $x$ and the Bayesian evidence $Z$ using $\Delta \chi_{\text {eff }}^{2}=-2 \ln p(x)-2 \ln Z$.

\begin{tabular}{c||c|c||cc||cc}
\multirow{2}{*}{ Dataset } & \multirow{2}{*}{ Case } & \multirow{2}{*}{ Prior type } & \multicolumn{2}{c||}{ NO } & \multicolumn{2}{c}{ IO } \\
& & & bestfit & $2 \sigma$ range & bestfit & $2 \sigma$ range \\
\hline \hline OSC & \multirow{2}{*}{$\mathrm{B}$} & $\operatorname{linear}$ & 0.33 & $0.05-1.25$ & 0.35 & $0.09-1.19$ \\
$+0 \nu \beta \beta$ & & $\log$ & 0.06 & $0.05-0.49$ & 0.10 & $0.09-0.44$ \\
\hline \hline OSC & \multirow{2}{*}{$\mathrm{B}$} & $\operatorname{linear}$ & 0.16 & $0.05-0.59$ & 0.19 & $0.09-0.53$ \\
$+\mathrm{CMB}$ & & $\log$ & 0.06 & $0.05-0.17$ & 0.10 & $0.09-0.18$ \\
\hline \hline \multirow{2}{*}{$\mathrm{ALL}$} & \multirow{2}{*}{$\mathrm{B}$} & $\operatorname{linear}$ & 0.13 & $0.05-0.58$ & 0.16 & $0.09-0.39$ \\
& & $\log$ & 0.06 & $0.05-0.16$ & 0.10 & $0.09-0.18$ \\
\hline
\end{tabular}

Table 6. Best fit values and marginalised $2 \sigma$ bounds for the sum of the neutrino masses (in $\mathrm{eV}$ ), for different datasets, using Case B and the priors shown in table 3. The case ALL corresponds to the $\mathrm{OSC}+\mathrm{CMB}+0 \nu \beta \beta$ combination.

the fiducial model. In the standard analysis (see e.g. [13-16, 36, 74]), the parameter space is usually scanned with a linear prior on the (degenerate) neutrino masses through their sum $\Sigma m_{\nu}$. In this section we shall show that the upper limits on $\Sigma m_{\nu}$ strongly depend on the prior choice. Therefore, special attention should be payed when using existing external results, computed by means of a given prior assumption, to derive constraints within analyses based on a different prior choice. 
Our constraints on $\Sigma m_{\nu}$ are summarised in table 6, where we show the best fit and the marginalised $2 \sigma$ limits for $\mathrm{NO}$ and IO, for different combinations of data sets, for the Case B and for different choices of priors, as detailed in section 2.2. Notice that we have not considered Case A since, as we will show below, it is much less efficient in exploring the parameter space with respect to Case B when considering oscillation data only. A quick inspection of table 6 tells us that the difference in the upper limits obtained when considering the same prior and data within normal and inverted neutrino mass ordering is not very large. ${ }^{8}$ This was expected, as these two cases are equivalent in the degenerate neutrino mass region.

Let us now focus on the crucial role of the prior choice (linear or logarithmic) which, according to table 6, has a critical impact on the total neutrino mass bounds. Sampling a variable with uniform probability in $\log x$ corresponds to assuming a probability $1 / x$ for $x$ itself, which leads to a bias in the scanning towards small values of $x$. As a consequence, the posterior of $\Sigma m_{\nu}$ is driven towards the smallest values allowed by oscillation data when the prior is logarithmic, because of the different available volume in the neutrino mass parameter space. Indeed, the upper bounds in the linear case are very close to those obtained previously by e.g. the Planck collaboration [36], while in the logarithmic case they are much smaller. Similar conclusions about the prior dependence of the results have been studied in Ref. [75], where it was shown that the proper way to treat constrained parameters (e.g. those forced to be non-negative by physical reasons) is to employ a Jeffreys prior, which is the one that maximises the sensitivity of the posterior distribution to data. The exact functional shape of the Jeffreys prior must be derived using the response of the likelihood to changes in the parameters. ${ }^{9}$ More recently, and based on the Lagrangian of the underlying particle physics theory (i.e. on the neutrino mass matrix), the theoretical priors on $\sum m_{\nu}$ have been extracted [76]. However, we do not implement none of the above prescriptions in computing our results, as (a) $\Sigma m_{\nu}$ is not a free parameter in our MCMC analyses, (b) the neutrino oscillation likelihood is multivariate in $\sin ^{2} \theta_{23}$, and (c) individual runs for the Dirac, Majorana and Majorana seesaw cases, which are beyond our main purposes, are mandatory while following the prescription of Ref. [76].

A final comment is devoted to the studies in the literature which employ the CMB posterior distributions obtained in the standard $\Lambda \mathrm{CDM}+\Sigma m_{\nu}$ model as a prior for other analyses. Let us firstly comment that the problem of the prior affecting the $\Sigma m_{\nu}$ bounds is a consequence of the fact that we adopt the mass of the lightest neutrino, which is unconstrained from below, as a parameter in our analyses. For standard cosmological analyses, since neutrino oscillations do constrain $\Sigma m_{\nu}$ from below, it is not necessary to consider a logarithmic prior on $\Sigma m_{\nu}$ and a linear one is sufficient, even though it may not be the more appropriate one [75] (see above). As demonstrated in table 6, the CMB is not powerful enough to constrain $\Sigma m_{\nu}[18]$ and therefore the posterior on this parameter depends heavily on the prior. Indeed, in Bayesian analysis, for a given data set, the posterior is the convolution of the prior with the likelihood. If the likelihood is sufficiently informative, the posterior has a very weak dependence on the prior. In the opposite case, data cannot provide sufficient information and the analysis returns something similar to what we knew before, i.e. the prior. Consequently, it is extremely dangerous to use a posterior distribution obtained previously with specific priors when performing an analysis that is based on different ones, because the internal consistency

\footnotetext{
${ }^{8}$ The only exception to this statement, appearing when using the linear prior and OSC $+\mathrm{CMB}+0 \nu \beta \beta$ data, is related to a statistical fluctuation that we found in the PolyChord output.

${ }^{9} \mathrm{~A}$ proper calculation of the Jeffreys prior should be performed separately for each of the parameterizations and priors we are adopting, a computation that is beyond the scope of this work.
} 
is lost. The situation might change in the future: if new data will have the power to constrain the mass of the lightest neutrino from below, the effect of the prior assumptions will vanish.

\subsection{Implications for the mass ordering}

We shall focus in the following on the preference for one neutrino mass ordering over the other from observations. Figure 3 illustrates the Bayes factors for NO over IO for different prior choices. First of all, we notice that the preference towards NO is solely driven by neutrino oscillation data, regardless of the selected priors. Indeed, when one simply considers the Bayesian analysis of the neutrino oscillation data and varies only the five mixing parameters (first point on the left, named as no mass scale), the preference $\ln \left(B_{\mathrm{NO}, \mathrm{IO}}\right) \simeq 2.5$ comes from the difference in the minimum $\Delta \chi^{2}$ from the global fit of neutrino oscillation measurements. In the Jeffreys' scale (table 1), this corresponds to a weak-to-moderate preference for NO.

Focusing on the first block of figure 3, which deals with neutrino oscillation data exclusively, one can notice that the choice of parameterization and prior has a direct effect on the strength of the preference of NO versus IO. While in the case of Case B the Bayes factors are highly stable against changes in the priors or in the allowed range for the lightest neutrino mass, for Case A the prior choice can strongly affect the Bayes factor in favour of NO. This happens because, within Case A, the volume of the parameter space that corresponds to the allowed values of the neutrino masses by oscillation data differs substantially from the total volume, sampled by means of the three individual neutrino masses, which are allowed to vary in a vast range. Within Case $\mathrm{B}$ this difference does not exist, because the parameter space is scanned using different variables, i.e. using informative priors. Since the difference between NO and IO mostly appears at small masses, prior choices that give more importance to small neutrino masses (i.e. logarithmic ones) will correspond to a stronger preference for NO within Case A. Figure 4 shows the posterior distribution for the three neutrino masses from neutrino oscillation data only, comparing NO and IO with linear (top panels) and logarithmic (bottom panels) priors. In the case of linear priors, the changes induced in the posterior volumes for the three mass eigenstates are nearly equivalent for $\mathrm{NO}$ and $\mathrm{IO}$, and therefore the Bayes factor will be very close to that extracted from oscillation data alone. However, as logarithmic priors naturally increase the importance of smaller masses, the difference in the posterior volumes for $\mathrm{NO}$ and $\mathrm{IO}$ is much more evident in this case, as can be seen in the lower panels of figure 4. The difference in the behaviour of $m_{1}$ and $m_{3}$ is practically irrelevant, as they reverse their roles in $\mathrm{NO}$ and IO. However, the change in the volume due to $m_{2}$ is crucial: Indeed, in the case of $\mathrm{NO} m_{2}$ is bounded from below by $\Delta m_{21}^{2}$, while in IO the lower bound is given by $\left|\Delta m_{31}^{2}\right|$. For this reason, the posterior volume in Case $\mathrm{A}$ strongly disfavours IO when a logarithmic prior is considered, and therefore Case A with logarithmic priors is the only one in which we find a strong preference for NO.

When we also account for information on the neutrino mass scale, either from CMB or $0 \nu \beta \beta$ probes, the situation for Case B does not change dramatically with respect to the oscillations-only case, as shown in figure 3. Case A is not considered because it is much less efficient than Case B (see below).

The Bayesian evidence analysis also opens the possibility to test the various parametrizations (i.e. Case A versus Case B) and prior choices (logarithmic versus linear). Figure 5 illustrates the Bayesian evidence of the different parameterizations and priors, normalised to the best scenario within each plot, for an easier comparison. Each of the panels corresponds to a given combination of data sets as indicated above and it is divided in two sub-panels, one for NO (left) and one for IO (right). 


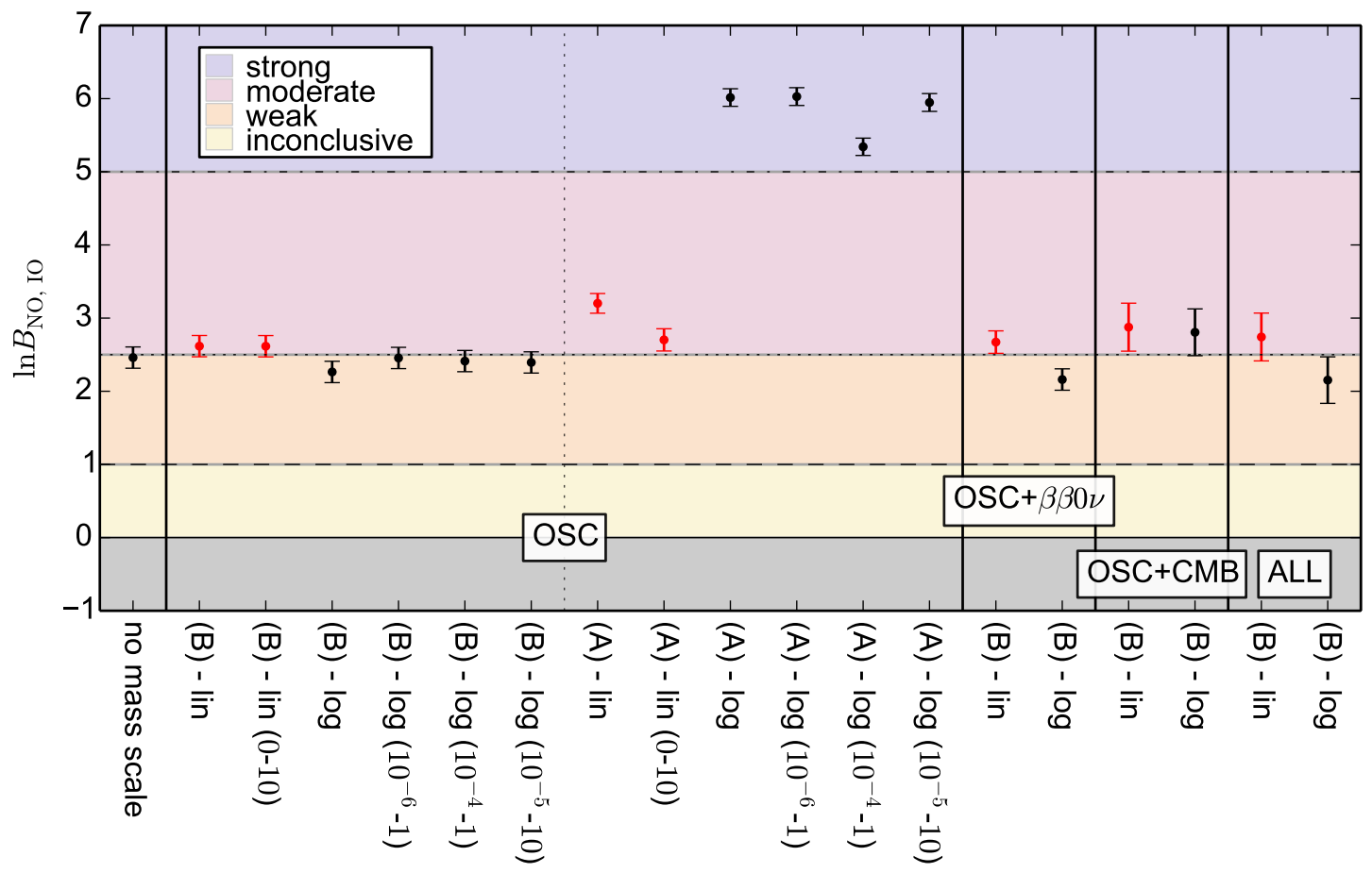

Figure 3. Graphical visualisation of the Bayesian factors comparing normal and inverted ordering. The horizontal lines indicate the values at which there is a change in the statistical significance of the evidence, according to the Jeffreys' scale (see table 1). Black (red) points indicate a logarithmic (linear) prior. The prior ranges are those reported in table 3 if not otherwise stated.

The top panel shows how a change in the prior ranges impacts the Bayesian evidences of Cases A and B, when either a linear or logarithmic prior is adopted and only oscillation measurements are considered in the numerical analysis. Notice that a different prior on the lightest neutrino mass leaves the Bayesian evidences of Case B unchanged both for NO and IO and for both linear and logarithmic priors. This is because the parameter $m_{\text {lightest }}$ remains unconstrained in all the cases, and the Bayes factor does not penalise unconstrained parameters through the Occam's razor. On the other hand, when considering Case A, the linear priors are always moderately-to-strongly less efficient for the parameter space exploration with respect to logarithmic priors, and the Case A itself is always much less efficient with respect to Case B. The reason is simple: the parametrization that uses three neutrino masses as free parameters corresponds to a waste of parameter space. Since neutrino oscillations determine the squared mass differences with a high accuracy, most of the parameter space in Case A at high neutrino masses is useless for the data fit, so that this parametrization is indeed penalised by the Occam's razor. Being motivated by the physical observables, Case B is therefore preferred over Case A when performing the analyses.

The bottom panels of figure 5 , which are restricted to Case $\mathrm{B}$, tell us that the addition of $0 \nu \beta \beta$ or cosmological data introduce a difference in the Bayesian evidences between linear and logarithmic priors. These data indeed show that the logarithmic priors are weakly-tomoderately more efficient, because in this latter case the fraction of volume corresponding to small masses, preferred by the data, is larger than in the linear case. 

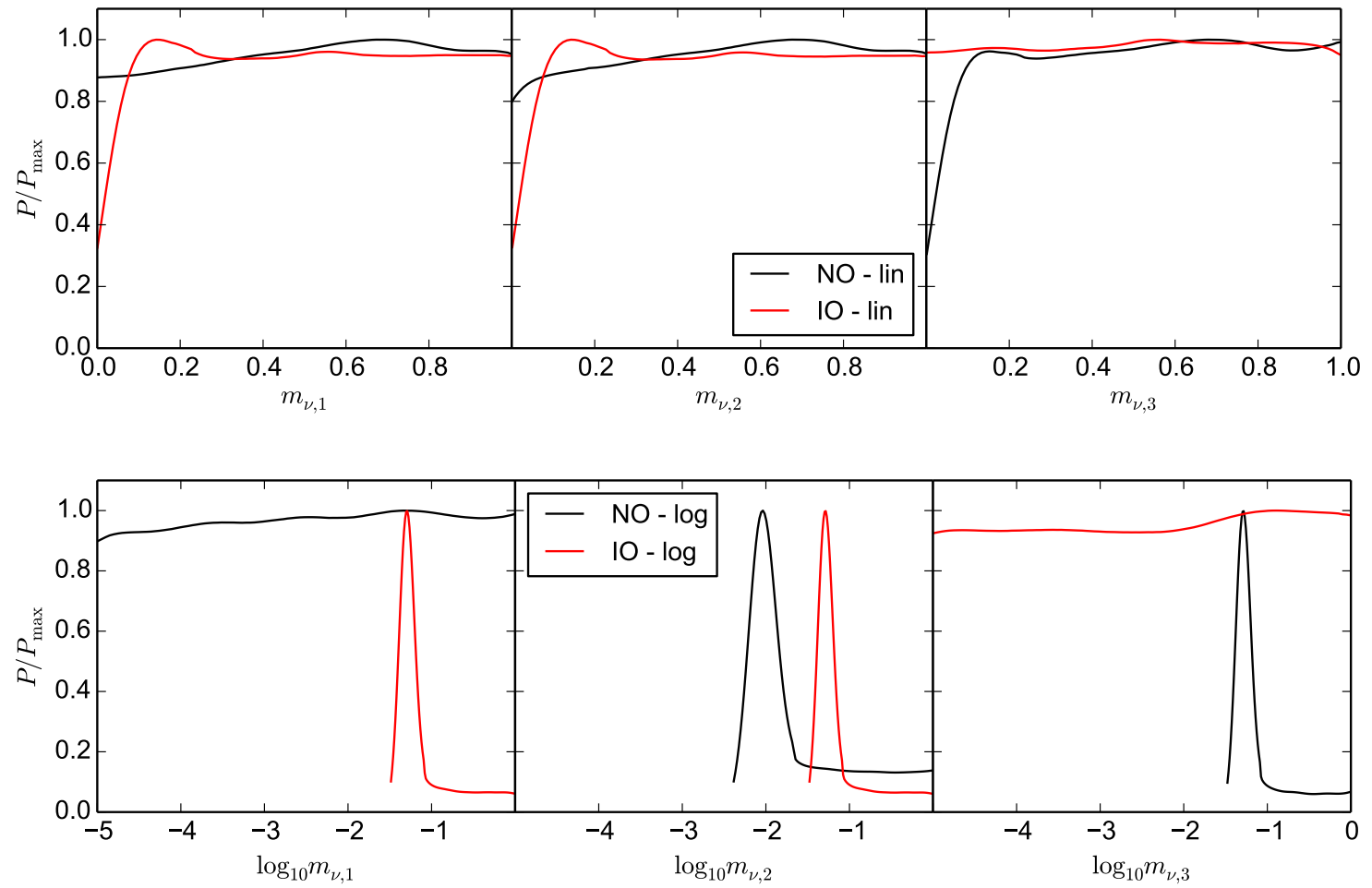

Figure 4. Difference in allowed volumes for the three absolute neutrino masses for NO and IO from neutrino oscillation data only. The top (bottom) panels show the case of linear (logarithmic) priors.

\section{Conclusions}

Plenty of work has been recently devoted in the literature to infer the neutrino mass ordering using a number of present observations [6, 16, 25-32], but a complete and self-consistent Bayesian analysis was still missing. Such an analysis is necessary in order to avoid strong claims in favour of normal mass ordering, based exclusively on the choice of priors. We have presented here the results obtained from the computationally expensive Bayesian evidence calculations, using current neutrino oscillation data, $0 \nu \beta \beta$ decay searches and Cosmic Microwave Background cosmological observations. In order to explicitly show the crucial role played by both the prior choice, we analyse two possible parametrizations: (a) Case A, in which the scan is performed over the individual neutrino masses $\left(m_{1}, m_{2}, m_{3}\right)$, and (b) Case $\mathrm{B}$, which is focused on the $\left(m_{\text {lightest }}, \Delta m_{21}^{2}, \Delta m_{31}^{2}\right)$ parameter space. For both parametrizations we study linear and logarithmic priors on the physical mass parameters, while we always use a linear prior for the squared mass differences.

Focusing first on our main goal, the Bayesian evidence against IO recently claimed [30] and extensively debated in Ref. [31], we find that the value of the Bayes factor is $\ln \left(B_{\mathrm{NO}, \mathrm{IO}}\right) \simeq$ 2.5 for almost all the possible parametrizations and prior choices. This value, which only points to weak evidence for NO, is entirely due to neutrino oscillation data. There is, however, one single combination in which we find strong evidence for NO, corresponding to the particular case on which Ref. [30] was focused on, i.e. when the scan is performed over the $\left(m_{1}, m_{2}, m_{3}\right)$ parameter space with logarithmic priors. This strong preference for NO arises 

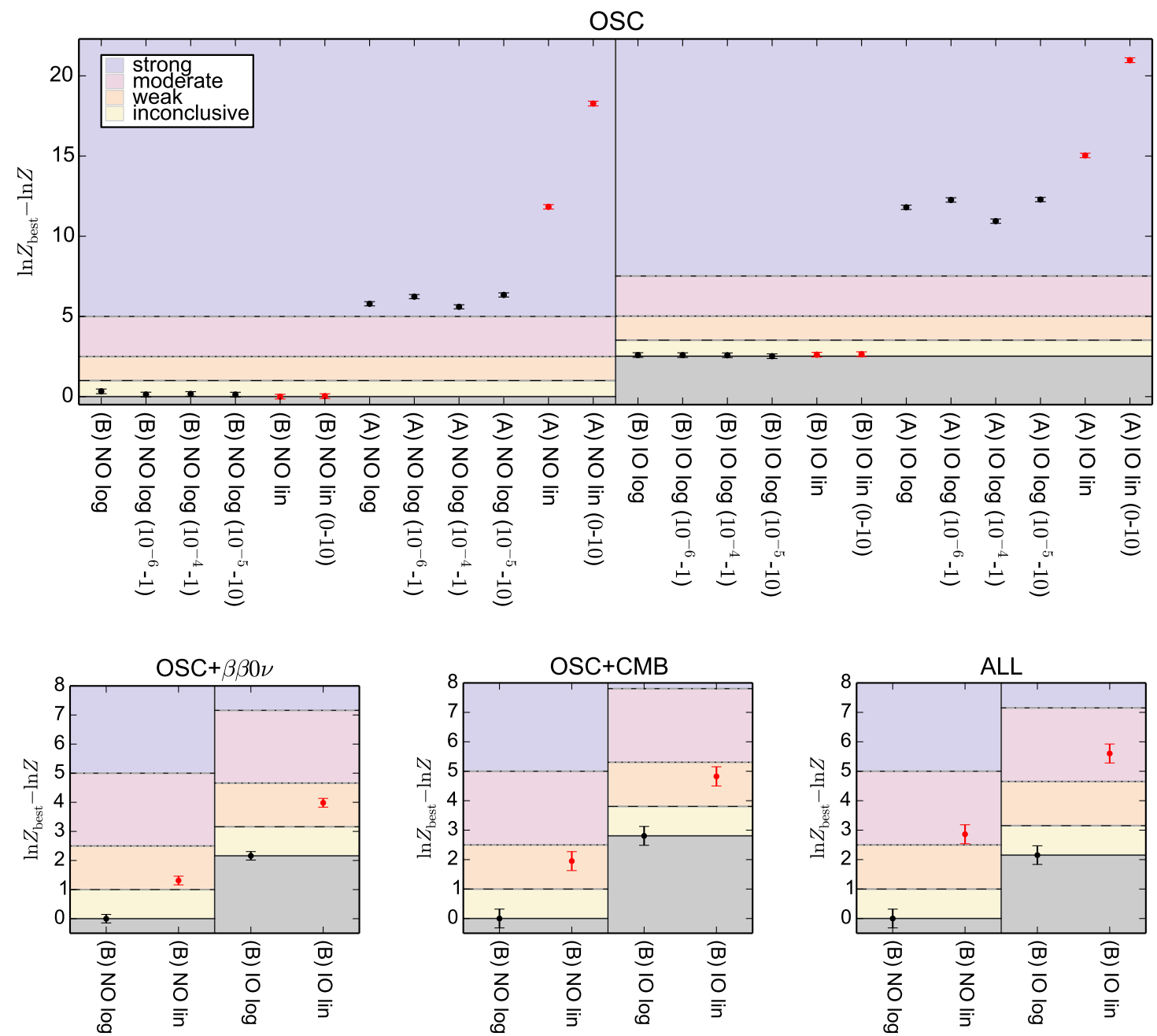

Figure 5. Graphical visualisation of the Bayesian evidences to compare the various neutrino mass parametrizations (Case A, only shown in the neutrino oscillation analyses, and Case B, shown for all the data combinations) and priors (logarithmic, in black, and linear, in red). Points are normalised with respect to the preferred Bayesian evidence $Z_{\text {best }}$ within each panel, which always corresponds to one of the Case B cases with NO. Colour codes are the same as in figure 3.

from the changes in the volume of $m_{2}$ between the two mass orderings, as this parameter is limited from below by $\Delta m_{21}^{2}$ in NO but by $\left|\Delta m_{31}^{2}\right|$ in IO. While our cosmological data sets are based on CMB measurements carried out by the Planck mission, we do not expect that our main conclusions change significantly when adding other possible observations, as Baryon Acoustic Oscillation (BAO) data. For instance, Ref. [26] quotes a modest 1.5:1 for the odds in favour of $\mathrm{NO}$ when $\mathrm{BAO}$ data is also considered in the analyses, corresponding to an uninformative $\ln B_{\mathrm{NO}, \mathrm{IO}} \simeq 0.4$. Consequently, our conclusions would be practically unchanged when adding the BAO information.

Another target of our study was the comparison between the Bayesian bounds on the neutrino mixing parameters to those obtained by means of frequentist approaches, finding, in general, a very good agreement. 
We have also shown that oscillation data strongly prefer Case B versus Case A, as a scan over the $\left(m_{\text {lightest }}, \Delta m_{21}^{2}, \Delta m_{31}^{2}\right)$ parameter space, being motivated by the physical observables, is more efficient than an analysis based on the three neutrino mass eigenstates. Furthermore, we have also computed the Bayesian evidence for logarithmic versus linear priors from current data, reporting that logarithmic priors guarantee a weakly-to-moderately more efficient scan of the parameter space.

The results derived here should be regarded as a guidance for future studies focused on the Bayesian comparison of the two neutrino mass orderings within the context of a global analysis involving neutrino oscillation, cosmological and neutrinoless double-beta decay data. Such studies should use the parametrization involving the neutrino squared mass splittings plus the lightest neutrino mass with a logarithmic prior. In addition, future studies should avoid using pre-computed limits on the total neutrino mass $\sum m_{\nu}$, since these can change dramatically when moving from one prior choice to another. The most general message of this paper is that Bayesian model comparison provides us with techniques that can be used in order to produce self-consistent and unbiased results. However, dedicated and careful analyses exploring all the different priors and parametrizations are always required in order to ensure robust and reliable conclusions.

\section{Acknowledgments}

We thank J. Lesgourgues and S. Pastor for very useful suggestions and improvements on the manuscript and to M. Hirsch and M. Sorel for fruitful discussions on neutrinoless double beta decay and the related nuclear matrix elements. Work supported by the Spanish grants FPA2014-57816-P, FPA2014-58183-P, FPA2017-85216-P, FPA2015-68783-REDT, FPA2017-85985-P and SEV-2014-0398 (MINECO), FPU13/03729 (MECD) and PROMETEOII/2014/050, PROMETEOII/2014/084 and GV2016-142 (Generalitat Valenciana). OM is also supported by the European Union's Horizon 2020 research and innovation program under the Marie Skłodowska-Curie grant agreements No. 690575 and 674896.

\section{References}

[1] A. B. McDonald, Nobel Lecture: The Sudbury Neutrino Observatory: Observation of flavor change for solar neutrinos, Rev. Mod. Phys. 88 (2016) 030502.

[2] T. Kajita, Nobel Lecture: Discovery of atmospheric neutrino oscillations, Rev. Mod. Phys. 88 (2016) 030501.

[3] J. Lesgourgues and S. Pastor, Massive neutrinos and cosmology, Phys. Rept. 429 (2006) 307-379, [astro-ph/0603494].

[4] J. Lesgourgues, G. Mangano, G. Miele and S. Pastor, Neutrino Cosmology. Cambridge University Press (CUP), 2013, 10.1017/cbo9781139012874.

[5] M. Lattanzi and M. Gerbino, Status of neutrino properties and future prospects - Cosmological and astrophysical constraints, 1712.07109.

[6] P. F. de Salas, D. V. Forero, C. A. Ternes, M. Tortola and J. W. F. Valle, Status of neutrino oscillations 2017, 1708.01186.

[7] DUNE collaboration, R. Acciarri et al., Long-Baseline Neutrino Facility (LBNF) and Deep Underground Neutrino Experiment (DUNE), 1512.06148.

[8] IceCube PINGU collaboration, M. G. Aartsen et al., Letter of Intent: The Precision IceCube Next Generation Upgrade (PINGU), 1401.2046. 
[9] KM3NET collaboration, S. Adrian-Martinez et al., Letter of intent for KM3NeT 2.0, J. Phys. G43 (2016) 084001, [1601.07459].

[10] J. Schechter and J. W. F. Valle, Neutrinoless Double beta Decay in SU(2) x U(1) Theories, Phys. Rev. D25 (1982) 2951.

[11] J. J. Gomez-Cadenas, J. Martin-Albo, M. Mezzetto, F. Monrabal and M. Sorel, The Search for neutrinoless double beta decay, Riv. Nuovo Cim. 35 (2012) 29-98, [1109.5515].

[12] S. Dell'Oro, S. Marcocci, M. Viel and F. Vissani, Neutrinoless double beta decay: 2015 review, Adv. High Energy Phys. 2016 (2016) 2162659, [1601.07512].

[13] N. Palanque-Delabrouille et al., Neutrino masses and cosmology with Lyman-alpha forest power spectrum, JCAP 11 (2015) 011, [1506.05976].

[14] E. Di Valentino, E. Giusarma, O. Mena, A. Melchiorri and J. Silk, Cosmological limits on neutrino unknowns versus low redshift priors, Phys. Rev. D93 (2016) 083527, [1511.00975].

[15] A. J. Cuesta, V. Niro and L. Verde, Neutrino mass limits: robust information from the power spectrum of galaxy surveys, Phys. Dark Univ. 13 (2016) 77-86, [1511.05983].

[16] S. Vagnozzi, E. Giusarma, O. Mena, K. Freese, M. Gerbino, S. Ho et al., Unveiling $\nu$ secrets with cosmological data: neutrino masses and mass hierarchy, Phys. Rev. D96 (2017) 123503, [1701.08172].

[17] M. Gerbino, M. Lattanzi and A. Melchiorri, $\nu$ generation: Present and future constraints on neutrino masses from global analysis of cosmology and laboratory experiments, Phys. Rev. D93 (2016) 033001, [1507.08614].

[18] M. Archidiacono, T. Brinckmann, J. Lesgourgues and V. Poulin, Physical effects involved in the measurements of neutrino masses with future cosmological data, JCAP 02 (2017) 052, [1610.09852].

[19] J. Hamann, S. Hannestad and Y. Y. Y. Wong, Measuring neutrino masses with a future galaxy survey, JCAP 11 (2012) 052, [1209.1043].

[20] S. Betts et al., Development of a Relic Neutrino Detection Experiment at PTOLEMY: Princeton Tritium Observatory for Light, Early-Universe, Massive-Neutrino Yield, in Proceedings, Community Summer Study 2013: Snowmass on the Mississippi (CSS2013): Minneapolis, MN, USA, July 29-August 6, 2013, 2013, 1307.4738.

[21] A. G. Cocco, G. Mangano and M. Messina, Probing low energy neutrino backgrounds with neutrino capture on beta decaying nuclei, JCAP 06 (2007) 015, [hep-ph/0703075].

[22] P. F. de Salas, S. Gariazzo, J. Lesgourgues and S. Pastor, Calculation of the local density of relic neutrinos, JCAP 09 (2017) 034, [1706.09850].

[23] J. Zhang and X. Zhang, Gravitational Clustering of Cosmic Relic Neutrinos in the Milky Way, 1712.01153.

[24] A. J. Long, C. Lunardini and E. Sabancilar, Detecting non-relativistic cosmic neutrinos by capture on tritium: phenomenology and physics potential, JCAP 08 (2014) 038, [1405.7654].

[25] S. Hannestad and T. Schwetz, Cosmology and the neutrino mass ordering, JCAP 11 (2016) 035, [1606.04691].

[26] M. Gerbino, M. Lattanzi, O. Mena and K. Freese, A novel approach to quantifying the sensitivity of current and future cosmological datasets to the neutrino mass ordering through Bayesian hierarchical modeling, Phys. Lett. B775 (2017) 239-250, [1611.07847].

[27] F. Capozzi, E. Di Valentino, E. Lisi, A. Marrone, A. Melchiorri and A. Palazzo, Global constraints on absolute neutrino masses and their ordering, Phys. Rev. D 95 (2017) 096014, [1703. 04471]. 
[28] S. Wang and D.-M. Xia, Constraints on the sum of neutrino masses from Bayesian analysis of the latest cosmological data, 1707.00588.

[29] I. Esteban, M. C. Gonzalez-Garcia, M. Maltoni, I. Martinez-Soler and T. Schwetz, Updated fit to three neutrino mixing: exploring the accelerator-reactor complementarity, JHEP 1701 (2017) 087, [1611.01514].

[30] F. Simpson, R. Jimenez, C. Pena-Garay and L. Verde, Strong Evidence for the Normal Neutrino Hierarchy, JCAP 06 (2017) 029, [1703.03425].

[31] T. Schwetz, K. Freese, M. Gerbino, E. Giusarma, S. Hannestad, M. Lattanzi et al., Comment on "Strong Evidence for the Normal Neutrino Hierarchy", 1703.04585.

[32] A. Caldwell, A. Merle, O. Schulz and M. Totzauer, A Global Bayesian Analysis of Neutrino Mass Data, Phys. Rev. D96 (2017) 073001, [1705.01945].

[33] M. Agostini et al., Background free search for neutrinoless double beta decay with GERDA Phase II, 1703.00570.

[34] EXO-200 collaboration, J. B. Albert et al., Search for Majorana neutrinos with the first two years of EXO-200 data, Nature 510 (2014) 229-234, [1402.6956].

[35] KamLAnD-Zen collaboration, A. Gando et al., Search for Majorana Neutrinos near the Inverted Mass Hierarchy Region with KamLAND-Zen, Phys. Rev. Lett. 117 (2016) 082503, [1605. 02889].

[36] Planck collaboration, P. A. R. Ade et al., Planck 2015 results. XIII. Cosmological parameters, Astron. Astrophys. 594 (2016) A13, [1502.01589].

[37] R. Trotta, Bayes in the sky: Bayesian inference and model selection in cosmology, Contemp. Phys. 49 (2008) 71-104, [0803.4089].

[38] W. J. Handley, M. P. Hobson and A. N. Lasenby, PolyChord: nested sampling for cosmology, Mon. Not. Roy. Astron. Soc. 450 (2015) L61-L65, [1502.01856].

[39] W. J. Handley, M. P. Hobson and A. N. Lasenby, PolyChord: next-generation nested sampling, Monthly Notices of the Royal Astronomical Society 453 (jun, 2015) 4384, [1506.00171].

[40] A. Lewis, A. Challinor and A. Lasenby, Efficient computation of CMB anisotropies in closed FRW models, Astrophys. J. 538 (2000) 473-476, [astro-ph/9911177].

[41] A. Lewis and S. Bridle, Cosmological parameters from CMB and other data: A Monte Carlo approach, Phys. Rev. D66 (2002) 103511, [astro-ph/0205436].

[42] C. Giunti and C. W. Kim, Fundamentals of Neutrino Physics and Astrophysics. 2007.

[43] A. Giuliani and A. Poves, Neutrinoless Double-Beta Decay, Adv. High Energy Phys. 2012 (2012) 857016.

[44] J. R. Pritchard and E. Pierpaoli, Constraining massive neutrinos using cosmological $21 \mathrm{~cm}$ observations, Phys. Rev. D78 (2008) 065009, [0805.1920].

[45] B. T. Cleveland, T. Daily, R. Davis, Jr., J. R. Distel, K. Lande, C. K. Lee et al., Measurement of the solar electron neutrino flux with the Homestake chlorine detector, Astrophys. J. 496 (1998) 505-526.

[46] F. Kaether, W. Hampel, G. Heusser, J. Kiko and T. Kirsten, Reanalysis of the GALLEX solar neutrino flux and source experiments, Phys. Lett. B685 (2010) 47-54, [1001.2731].

[47] SAGE collaboration, J. N. Abdurashitov et al., Measurement of the solar neutrino capture rate with gallium metal. III: Results for the 2002-2007 data-taking period, Phys. Rev. C80 (2009) 015807, [0901.2200].

[48] Super-Kamiokande collaboration, J. Hosaka et al., Solar neutrino measurements in super-Kamiokande-I, Phys. Rev. D73 (2006) 112001, [hep-ex/0508053]. 
[49] Super-Kamiokande collaboration, J. P. Cravens et al., Solar neutrino measurements in Super-Kamiokande-II, Phys. Rev. D78 (2008) 032002, [0803.4312].

[50] Super-Kamiokande collaboration, K. Abe et al., Solar neutrino results in Super-Kamiokande-III, Phys. Rev. D83 (2011) 052010, [1010.0118].

[51] Y. Nakano, "PhD Thesis, University of Tokyo." http://www-sk.icrr.u-tokyo.ac.jp/sk/_pdf/articles/2016/doc_thesis_naknao.pdf, 2016.

[52] SNO collaboration, B. Aharmim et al., An Independent Measurement of the Total Active B-8 Solar Neutrino Flux Using an Array of He-3 Proportional Counters at the Sudbury Neutrino Observatory, Phys. Rev. Lett. 101 (2008) 111301, [0806.0989].

[53] SNO collaboration, B. Aharmim et al., Low Energy Threshold Analysis of the Phase I and Phase II Data Sets of the Sudbury Neutrino Observatory, Phys. Rev. C81 (2010) 055504, [0910.2984].

[54] Borexino collaboration, G. Bellini et al., Final results of Borexino Phase-I on low energy solar neutrino spectroscopy, Phys. Rev. D89 (2014) 112007, [1308.0443].

[55] KamLAND collaboration, A. Gando et al., Constraints on $\theta_{13}$ from A Three-Flavor Oscillation Analysis of Reactor Antineutrinos at KamLAND, Phys. Rev. D83 (2011) 052002, [1009.4771].

[56] DAYA BAy collaboration, F. P. An et al., Measurement of electron antineutrino oscillation based on 1230 days of operation of the Daya Bay experiment, Phys. Rev. D 95 (2017) 072006, [1610.04802].

[57] RENO collaboration, J. H. Choi et al., Observation of Energy and Baseline Dependent Reactor Antineutrino Disappearance in the RENO Experiment, Phys. Rev. Lett. 116 (2016) 211801, [1511.05849].

[58] Double Chooz collaboration, Y. Abe et al., Improved measurements of the neutrino mixing angle $\theta_{13}$ with the Double Chooz detector, JHEP 1410 (2014) 086, [1406.7763].

[59] Super-Kamiokande collaboration, R. Wendell et al., Atmospheric neutrino oscillation analysis with sub-leading effects in Super-Kamiokande I, II, and III, Phys. Rev. D81 (2010) 092004, [1002.3471].

[60] ICECuBE collaboration, M. G. Aartsen et al., Determining neutrino oscillation parameters from atmospheric muon neutrino disappearance with three years of IceCube DeepCore data, Phys. Rev. D91 (2015) 072004, [1410.7227].

[61] ICECUBE collaboration, "IceCube Oscillations: 3 years muon neutrino disappearance data." https://icecube.wisc.edu/science/data/nu_osc, October, 2016.

[62] ANTARES collaboration, S. Adrian-Martinez et al., Measurement of Atmospheric Neutrino Oscillations with the ANTARES Neutrino Telescope, Phys. Lett. B714 (2012) 224-230, [1206.0645].

[63] ICECuBe collaboration, M. G. Aartsen et al., Measurement of Atmospheric Neutrino Oscillations at 6-56 GeV with IceCube DeepCore, 1707.07081.

[64] Super-Kamiokande collaboration, K. Abe et al., Atmospheric neutrino oscillation analysis with external constraints in Super-Kamiokande I-IV, 1710.09126.

[65] K2K collaboration, M. H. Ahn et al., Measurement of Neutrino Oscillation by the K2K Experiment, Phys. Rev. D74 (2006) 072003, [hep-ex/0606032].

[66] MINOS collaboration, P. Adamson et al., Measurement of Neutrino and Antineutrino Oscillations Using Beam and Atmospheric Data in MINOS, Phys. Rev. Lett. 110 (2013) 251801, [1304.6335]. 
[67] MINOS collaboration, P. Adamson et al., Combined analysis of $\nu_{\mu}$ disappearance and $\nu_{\mu} \rightarrow \nu_{e}$ appearance in MINOS using accelerator and atmospheric neutrinos, Phys. Rev. Lett. 112 (2014) 191801, [1403.0867].

[68] K. Abe et al., Updated T2K measurements of muon neutrino and antineutrino disappearance using 1.5e21 protons on target, Phys. Rev. D96 (2017) 011102, [1704.06409].

[69] T2K collaboration, K. Abe et al., First combined analysis of neutrino and antineutrino oscillations at T2K, Phys. Rev. Lett. 118 (2017) 151801, [1701.00432].

[70] The NOvA collaboration, P. Adamson et al., Measurement of the neutrino mixing angle $\theta_{23}$ in NOvA, Phys. Rev. Lett. 118 (2017) 151802, [1701.05891].

[71] P. Adamson et al., Constraints on oscillation parameters from $\nu_{e}$ appearance and $\nu_{\mu}$ disappearance in NOvA, Phys. Rev. Lett. 118 (2017) 231801, [1703.03328].

[72] Planck collaboration, R. Adam et al., Planck 2015 results. I. Overview of products and scientific results, Astron. Astrophys. 594 (2016) A1, [1502.01582].

[73] Planck collaboration, N. Aghanim et al., Planck 2015 results. XI. CMB power spectra, likelihoods, and robustness of parameters, Astron. Astrophys. 594 (2016) A11, [1507.02704].

[74] E. Giusarma, M. Gerbino, O. Mena, S. Vagnozzi, S. Ho and K. Freese, Improvement of cosmological neutrino mass bounds, Phys. Rev. D94 (2016) 083522, [1605.04320].

[75] S. Hannestad and T. Tram, Optimal prior for Bayesian inference in a constrained parameter space, 1710.08899 .

[76] A. J. Long, M. Raveri, W. Hu and S. Dodelson, Neutrino Mass Priors for Cosmology from Random Matrices, 1711.08434. 